\title{
Recherches sur les Grégarines (Gregarinida: Stenophoridae) du Diplopode troglobie Typhloiulus bureschi Verhoeff en Bulgarie
}

\author{
par \\ Vassil GOLEMANSKY et Dimitar TASCHEV*
}

INTRODUCTION

Typhloiulus bureschi Verhoeff, 1926 (Diplopoda: Julidae) est un troglobie typique, habitant plusieurs grottes bulgares dans la région de la montagne Stara planina (le Balkan). Il se trouve toujours dans les zones profondes des grottes, errant sur l'argile humide ou sur le guano de chauves-souris (Guéorguiev et Beron, 1962; Strasser, 1966). Il semble que Typhloiulus bureschi est aussi une espèce endémique dans la faune de Bulgarie (Guéorguiev, 1966).

Nos premières recherches sur les parasites unicellulaires de Typhloiulus bureschi de la grotte "Temnata doupka", près de la gare "Lakatnik", faites en 1959, ont montré que l'animal est habité par deux espèces de grégarines du genre Stenophora Labbé qui se développent dans l'intestin de l'hôte. Nos recherches ultérieures sur la parasitofaune de cette espèce, faites en 1968,1970, 1971 et 1972, nous ont permis de traiter un matériel plus riche et de ramasser plus de données sur la morphologie et la taxonomie des eugrégarines trouvées, l'une desquelles s'est avérée une espèce nouvelle pour la sicience. Dans le présent travail on fera une description plus détaillée des grégarines trouvées dans Typhloiulus bureschi, qui sont d'un grand intérêt parasitologique, aussi bien que spéléologique.

\section{RÉSULTATS}

Stenophora typhloiuli n.sp. Pl. I, fig. 1-9, Pl. II, fig. 4a, 5a.

DESCRIPTION: Les jeunes trophozoïtes, détachés de l'épithélium de l'intestin, sont de forme cylindrique allongée, dont l'extrémité antérieure est faiblement rétrécie (Pl. I, fig. 1; Pl. II, fig. 4a). La cloison entre le proto- et le deutomérite, chez les plus jeunes trophozoites, n'est pas bien marquée. Le deutomérite, incolore et transparent, se termine par un retranchement à l'extrémité postérieure.

Le proto- et le deutomérite des trophozoites à dimensions de plus de $40 \mu$ sont déjà bien différenciés. Le protomérite a la forme d'un cône tronqué, court, dont les côtés sont convexes et il est plus large que long. Cette forme et le rapport longueur: largeur du protomérite, qui varie de 1:1,2 à 1:1,9, restent les mêmes dans tous les

\footnotetext{
* Institut Zoologique \&. Musée, 1, boul. Rouski, Sofia/Bulgarie.
} 


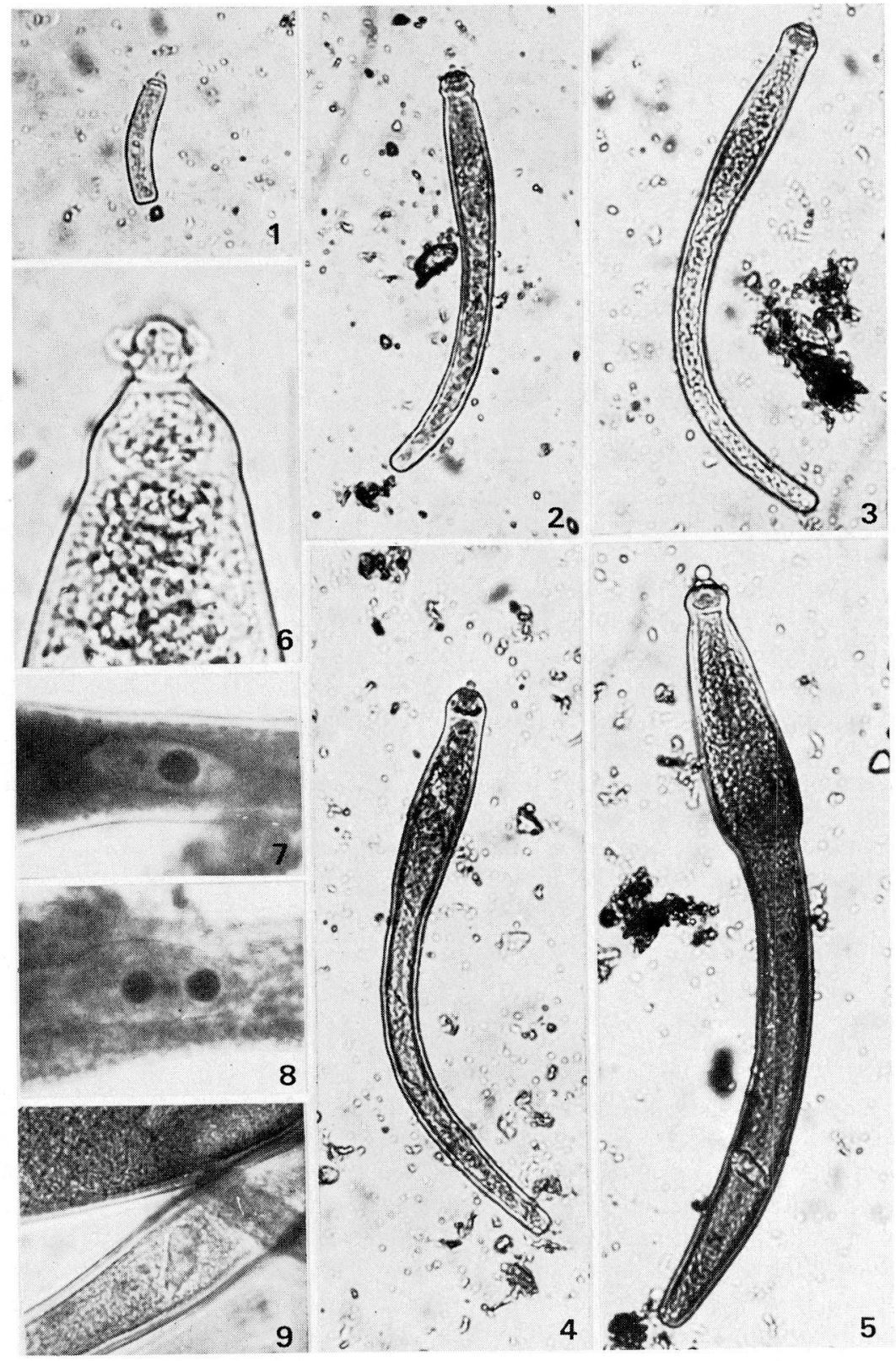


stades ultérieurs du développpement de l'espèce. Le proto- et le deutomérite sont séparés par une couche fine de cytoplasme hyalin.

L'épimérite est rarement observé dans les populations de Stenophora typhloiuli n.sp. et il a la forrne typique du genre - un bouton à diamètre de 5-7 $\mu$ (Pl. I, fig. 4,5). Souvent une partie du paroi de la cellule épithéliale reste sur lui (Pl. I, fig. 6; Pl. II, fig. 4a).

$\mathrm{Au}$ cours de l'accroissement des trophozoïtes, le deutomérite de Stenophora typhloiuli n.sp. s'élargit progressivement et de cylindrique il devient fusiforme allongé. Dans des populations de différents individus de l'hôte, il garde sa forme cylindrique chez les exemplaires longs de 100 à $120 \mu$; au-delà de cette limite, dans le premier tiers ces animaux, l'accumulation de paraglycogène augmente et le deutomérite acquiert une nouvelle forme caractéristique (Pl. I, fig. 5; Pl. II, fig. 5a). En même temps, ces changements subissent aussi les valeurs de l'indice WD:LT qui varient de 1:2,2 - 1:4,7 chez les plus jeunes trophozoïtes jusqu'à 1:7 - 1:11 chez les formes nématoides les plus longues. Chez les exemplaires adultes, où l'accumulation de paraglycogène dans le premier tiers du deutomérite a déjà commencé, elles varient de $1: 5-1: 9,4$.

Le nucleus des jeunes trophozoites est sphérique ou large ovale, avec un karyosome central et il est situé dans la partie centrale du déutomérite. Avec l'accroissement des dimensions des grégarines il devient allongé ellipsoïdal et parfois il a plus d'un karyosome (P1. I, fig. 7,8). Chez quelques-uns des trophozoïtes que nous avons observés, à dimensions de plus de $300 \mu$, le nucleus perd sa forme régulière et fait preuve de certains signes de dégénération (Pl. I, fig. 9). Chez les trophozoïtes adultes, le nucleus se trouve d'habitude à la frontière de la partie élargie et de la partie cylindrique du deutomérite.

D'après nos observations, l'espèce atteint les dimentsions maxima de $360 \mu$. La largeur maximum des individus, observés in vivo et immédiatement après la dissection de l'hôte, ne dépasse pas à l'ordinaire 40-45 $\mu$ dans la partie la plus large. Les dimensions d'une partie des trophozoïtes observés, ainsi que leurs changements au cours de l'accroissement et du développement sont présentés au Tableau 1.

Stenophora typhloiuli n.sp. est un parasite ordinaire et on le trouve dans la plupart des individus de T.bureschi que nous avons observés. L'intensité d'invasion que nous avons observée a un large diapason de variations: de parasites isolés à de dizaines d'exemplaires dans un individu.

On n'a pas observé des kustes et des spores.

HÔTE: Typhloiulus bureschi Verhoeff, 1926 (Diplopoda, Nematophora, Julidae). LOCALITES: 1. La grotte "Temnata doupka", près de la gare Lakatnik, district de Sofia. 12.4.1959, 17.2.1968, 8.10.1970, 12.7.1971, 4.7.1972.

2. La grotte "Ledenika", district de Vratza, 18.2.1968, 30.5.1972.

\section{Planche 1.}

Fig. 1-5. Une série de cinq stades succéssifs de Stenophora typhloiuli n.sp.

Fig. 6. Détail de l'épimérite de St. typhloiuli n.sp.

Fig. 7,8. Noyaux typiques des exemplaires adultes de St. typhloiuli n.sp.

Fig. 9. Noyau en dégénération chez un individu adulte de St. typhloiuli n.sp. 


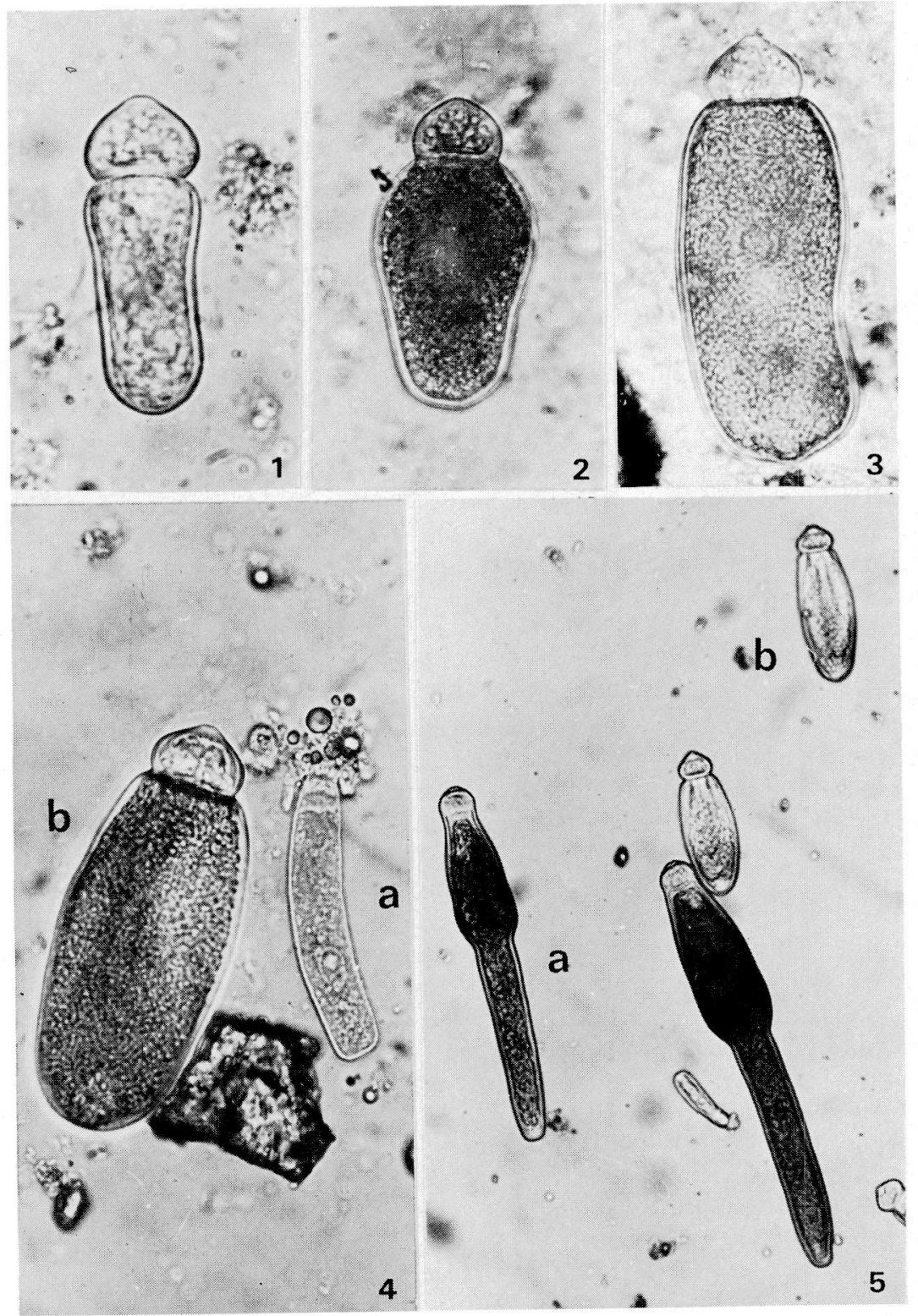

Planche 2.

Fig. 1-3. Une série de trois stades succéssifs de Stenophora sp.

Fig. 4. Jeune trophozoïte de St. typhloiuli n.sp. (a) et trophozoïte adulte de Stenophora sp.

Fig. 5. Deux trophozoïtes adultes de St. typhloiuli n.sp. (a) et de Stenophora sp. (b). 
Tableau 1: Tableau des dimensions des trophozoïtes de Stenophora typhloiuli n.sp. et leus changements lors de l'accroissement*

\begin{tabular}{|c|c|c|c|c|c|c|c|c|c|c|c|}
\hline LT & $\begin{array}{l}\text { Ep } \\
\mathrm{L}\end{array}$ & $\begin{array}{l}\text { erit } \\
\text {. }\end{array}$ & $\begin{array}{l}\text { roton } \\
\text { LP }\end{array}$ & $\begin{array}{l}\text { hérite } \\
\text { WP }\end{array}$ & $\begin{array}{l}\text { Deuto } \\
\text { LD }\end{array}$ & $\begin{array}{l}\text { mérite } \\
\text { WD }\end{array}$ & LP:WT & WD:LT & WP:WD & LP:WP & Nucleus \\
\hline 21 & - & - & - & - & - & 9,5 & - & $1: 2,2$ & - & - & - \\
\hline 39 & - & - & 6,6 & 8,4 & 32,4 & 11 & $1: 5,9$ & $1: 3,5$ & $1: 1,3$ & $1: 1,3$ & - \\
\hline 49 & 5 & 5 & 5 & 8 & 44 & 10,5 & $1: 9,8$ & $1: 4,7$ & $1: 1,3$ & $1: 1,6$ & - \\
\hline 60 & 5 & 6 & 6 & 7 & 54 & 14 & $1: 10,0$ & $1: 4,3$ & $1: 2,0$ & $1: 1,2$ & - \\
\hline 84 & - & - & 6 & 10,5 & 78 & 13 & $1: 14,0$ & $1: 6,5$ & $1: 1,2$ & $1: 1,8$ & - \\
\hline 127 & 6 & 6 & 11 & 13 & 116 & 21 & $1: 11,5$ & $1: 6,0$ & $1: 1,6$ & $1: 1,2$ & - \\
\hline 165 & - & - & 15 & 18 & 150 & 20 & $1: 11,0$ & $1: 8,3$ & $1: 1,1$ & $1: 1,2$ & oval, $13 \times 6$ \\
\hline 182 & - & - & 14 & 19 & 168 & 31 & $1: 13,0$ & $1: 5,9$ & $1: 1,6$ & $1: 1,4$ & - \\
\hline 197 & 7 & 7 & 12 & 16 & 185 & 25 & $1: 16,4$ & $1: 7,9$ & $1: 1,6$ & $1: 1,3$ & oval, $17 \times 8$ \\
\hline 211 & - & - & 11 & 16 & 200 & 26 & $1: 19,2$ & $1: 8.1$ & $1: 1,6$ & $1: 1,5$ & - \\
\hline 244 & - & - & 12 & 17 & 232 & 26 & $1: 20,3$ & $1: 9,4$ & $1: 1,5$ & $1: 1,4$ & - \\
\hline 264 & - & - & 14 & 19 & 250 & 38 & $1: 18,9$ & $1: 6,9$ & $1: 2,0$ & $1: 1,4$ & - \\
\hline 299 & - & - & 14 & 19 & 285 & 34 & $1: 21,4$ & $1: 8,8$ & $1: 1,8$ & $1: 1,4$ & - \\
\hline 320 & - & - & 14 & 22,5 & 306 & 35 & $1: 22,9$ & $1: 9,1$ & $1: 1,6$ & $1: 1,6$ & oval, $28 \times 15$ \\
\hline 356 & & - & 15 & 28 & 341 & 44 & $1: 23,7$ & $1: 8,1$ & $1: 1,6$ & $1: 1,9$ & oval, $28 \times 15$ \\
\hline
\end{tabular}

* Toutes les dimensions sont données en microns

$\begin{array}{lll}\text { LT } & - & \text { longueur totale du corps } \\ \text { L } & - & \text { longueur } \\ \text { W } & - & \text { largeur } \\ \text { LP } & - & \text { longueur du protomérite } \\ \text { LD } & - & \text { longueur du deutomérite } \\ \text { WP } & - & \text { largeur du protomérite } \\ \text { WD } & - & \text { largeur du deutomérite }\end{array}$

MATÉRIEL: 7 préparations microscopique dans la collection de l'INstitut Zoologique et Musée près l'Académie bulgare des Sciences, Sofia.

DISCUSSION: Par la forme du corps, Stenophora typhloiuli n.sp. présente quelques ressemblances uniquement avec les espèces Stenophora bulgarosomae Golemansky, décrite du Diplopode cavernicole Bulgarosoma bureschi Verhoeff de Bulgarie (Golemansky, in litt.) et Stenophora nematoïdes Léger et Duboscq, trouvée dans Strongylosoma italicum Latzel des environ de Bastia, Corsica (Léger et Duboscq, 1903). Les deux espèces susmensionnées sont de forme nématoïde allongée et appartiennent aux. espèces du genre Stenophora sans papille ou pore au protomérite.

La nouvelle espèce, décrite par nous, diffère de St.bulgarosoma par la présence d'un élargissement fusiforme typique du deutomérite, par le caractère du protomérite et par le type du nucleus. Les dimensions de St.bulgarosomae sont aussi sensiblement plus grandes (plus de 500) que celles des trophozoïtes de St.typhloiuli n.sp. 
Stenophora typhloiuli n.sp. diffère de St.nematoïdes surtout par le type du protomérite. Chez la nouvelle espèce le protomérite a la forme typique d'un cône tronqué, court, dont les côtés sont convexes, tandis que chez St.nematoïdes il est de forme cylindrique. Comme om voit de la figure de Léger et Dubiscq (1903), le protomérite de St.nematoïdes est sensiblement plus long que large, tandis que chez St.typhloiuli n.sp., dans tous les cas observés, sa largeur est plus grande que la hauteur ( $\mathrm{LP}: \mathrm{WP}=1: 1,2-1: 1,9)$. Exepté le type du protomérite, les deux dernières espèces diffèrent aussi sensiblement par la morphologie du deutomérite, qui chez Stenophora nematoïdes a une configuration nématoïde mieux marquée. D’après Léger et Duboscq (1903) les plus grandes formes nématoïdes de St.nematoïdes ont des dimensions de $170 \times 7 \mu(\mathrm{LT}: \mathrm{WD}=1: 24)$, tandis que les formes allongées correspondantes de St.typhloiuli n.sp. ont des dimensions de $100-120 \mu$ et une largeur de 9 - $14 \mu$. Le rapport WD:LT varie dans les limites de 1:2,2 à 1:6,5.

Stenophora sp. (Pl. II, fig. 1,2,3,4b,5b).

Le corps des jeunes trophozoïtes de Stenophora sp. est allongé, presque cylindrique, avec un protomérite relativement grand et bien déterminé. Il a la forme d'un cône court et large, dont les angles postérieurs sont arrondis. La cloison entre le protomérite et le deutomérite est bien déterminée et marquée par une invagination profonde entre les deux parties du corps. A son extrémité apicale le protomérite est dépourvu de papille ou de pore (Pl. II, fig. 1).

Chez les jeunes trophozoïtes, le deutomérite est faiblement élargi dans le premier tiers du corps, où se trouve le nucleus sphérique. Son extrémité postérieure est arrondie (Pl. II, fig. 1,2).

Chez les stades ultérieurs du développpement de l'espèce, l'accroissement se fait au dépens du deutomérite et il en résulte que l'indice LP:LT augmente de 1:27 chez les plus petits individus jusqu'à 1:9 chez les plus grands. Le protomérite garde sa forme et reste toujours plus large que haut. Le rapport LP:WP reste constant et les limites de ses variations sont petites - de 1:1,2 à 1:1,7. On n'a pas observé d'épimérite.

Chez les trophozoïtes adultes, le deutomérite garde généralement sa forme cylindrique, mais à la suite de l'accumulation des réserves alimentaires, il devient plus épais et opaque. Le nucleus garde sa forme ronde et se trouve généralement dans la partie postérieure du deutomérite. La cuticule du corps est épaisse $(2,5-3 \mu)$ et pourvue de striations longitudinales profondes.

Les dimensions maxima des trophozoïtes de Stenophora $s p$. sont sensiblement plus petites que celles de Stenophora typhloiuli n.sp. Leurs valeurs et leurs changements au cours de l'accroissement des trophozoïtes sont présentés au Tableau 2. 
Tableau 2: Tableau des dimensions des trophozoïtes de Stenophora sp. et leurs changements lors de l'accroissement*

\begin{tabular}{|c|c|c|c|c|c|c|c|c|c|c|c|}
\hline \multirow{2}{*}{ LT } & \multicolumn{2}{|c|}{ Epimérite } & \multicolumn{2}{|c|}{ Protomérite } & \multicolumn{2}{|c|}{ Deutomérite } & \multirow{2}{*}{ LP:LT } & \multirow{2}{*}{ WD: LT } & \multirow{2}{*}{ WP:WD } & \multirow{2}{*}{ LP:WP } & \multirow{2}{*}{ Nucleus } \\
\hline & $\mathrm{L}$ & W & LP & WP & LD & WD & & & & & \\
\hline 1 & 2 & 3 & 4 & 5 & 6 & 7 & 8 & 9 & 10 & 11 & 12 \\
\hline 28 & - & - & 10,5 & 15,0 & 17,5 & 18,0 & $1: 2,7$ & $1: 1,6$ & $1: 1,2$ & $1: 1,4$ & - \\
\hline 47 & - & - & 12,0 & 18,0 & 35,0 & 15,0 & $1: 3,9$ & $1: 3,1$ & $1: 0,83$ & $1: 1,5$ & rond \\
\hline 66 & - & - & 15,0 & 19,0 & 51,0 & 22,0 & $1: 4,4$ & $1: 3,0$ & $1: 1,2$ & $1: 1,3$ & rond \\
\hline 74 & -- & - & 10,5 & 15,5 & 63,5 & 37,0 & $1: 7,0$ & $1: 2$ & $1: 2,4$ & $1: 1,5$ & - \\
\hline 81 & - & - & 15 & 21 & 66,0 & 22,2 & $1: 5,4$ & $1: 3,6$ & $1: 1,1$ & $1: 1,4$ & rond \\
\hline 84 & - & - & 15,5 & 19,0 & 68,5 & 35,5 & $1: 5,4$ & $1: 2,4$ & $1: 1,9$ & $1: 1,2$ & - \\
\hline 95 & - & - & 10,5 & 18,0 & 84,5 & 27,0 & $1: 9,0$ & $1: 3,5$ & $1: 1,5$ & $1: 1,7$ & - \\
\hline 100 & - & - & 15,5 & 18,0 & 84,5 & 46,0 & $1: 6,5$ & $1: 2,2$ & $1: 2,6$ & $1: 1,2$ & - \\
\hline 108 & - & - & 15,0 & 20,0 & 93,0 & 33,0 & $1: 7,2$ & $1: 3,3$ & $1: 1,7$ & $1: 1,3$ & - \\
\hline 109 & - & - & 15,0 & 21,0 & 94,0 & 39,0 & $1: 7,3$ & $1: 2,8$ & $1: 1,9$ & $1: 1,4$ & - \\
\hline 114 & - & - & 15,0 & 21,0 & 99,0 & 39,0 & $1: 7,6$ & $1: 2,9$ & $1: 1,9$ & $1: 1,4$ & - \\
\hline 120 & - & - & 14,0 & 21,0 & 106,0 & 32,0 & $1: 8,6$ & $1: 3,8$ & $1: 1,5$ & $1: 1,5$ & - \\
\hline
\end{tabular}

* Toutes les dimensions sont donnés en microns

LT - longueur totale du corps
L - longueur
W - largeur
LP - longueur du protomérite
LD - longueur du deutomérite
WP - largeur du protomérite
WD - largeur du deutomérite

Nos études ont montré que Stenophora $s p$. est une espèce qu'on peut trouver plus rarement que Stenophora typhloiuli n.sp. On a observé des invasions individuelles, aussi bien que mixtes.

HÔTE: Typhloiuli bureschi Verhoeff, 1926 (Diplopoda, Nematophora, Julidae).

LOCALISATION l'intestin.

LOCALITÉS: 1. La grotte "Temnata doupka", près de la gare Lakatnik, district de Sofia. 12.4.1959, 17.2.1968, 8.10.1970, 12.7.1971, 4.7.1972.

2. La grotte "Ledenika", district de Vratza. 18.2.1968, 30.5.1972.

MATERIEL: 5 préparations microscopiques dans la collection de l'Institut Zoologique et Musée près l'Académie bulgare des Sciences, Sofia.

DISCUSSION: L'identification de Stenophora sp. a été assez difficile, car les stades du développement du parasite que nous avons trouvés, présentent certains ressemblances morphologiques avec quelques autres espèces de Stenophora, décrites de différent Diplopodes épigés, tels que Stenophora strongilosomae Lipa de Strongilosoma pallipes (Olivier) et Stenophora orthomorphae Lipa d'Orthomopha gracilis (Koch), décrites de la Pologne (Lipa, 1967), les formes allongées de Stenophora chordeume Léger et Duboscq de Chordeuma silvestre $C$. Koch de la France (Léger et Duboscq, 1904), Stenophora fontaria (Crowley) Watson de Polydesmus sp. et Fontaria sp. de l'Amérique (Watson, 1916) et d'autres. 
Le manque d'observations plus complètes sur les autres stades du cycle vital de Stenophora $s p$. ne nous permet pas, dans le moment présent, de tirer une conclusion plus précise sur l'appartenance d'espèce du parasite que nous avons trouvé. De plus que, quoiqu'une espèce troglobie, il est bien possible que, par les eaux épigées ou par un autre moyen, les individus de Typhloiulus bureschi, habitant les grottes, soient invasés par les spores apportées du dehors de quelques-unes des espèces du genre Stenophora, habitant les Diplopodes épigés, et que les trophozoïtes de Stenophora sp. s'y développent. Malheureusement, l'identification a été entravée aussi par le fait que, dans la littérature parasitologique, la description d'une grande partie des espèces des grégarines des diplopodes, connues jusqu'à présent, est incomplète et réduite, ce qui fait possible les fautes lors de la détermination de la position taxonomique des eseces trouvées et l'éclaircissement de la systématique du genre Stenophora en général.

\section{RESUME}

Etude de la parasito-faune et plus particulièrement des Grégarines d'un Diplopode troglobie Typhloiulus bureschi Verhoeff, Iulide des grottes bulgares. Données sur la morphologie et la taxonomie des Eugrégarines trouvées chez ce Iulidae. Description d'une espèce nouvelle, Stenophora typhloiuli n.sp. et comparaison avec d'autres espèces du genre Stenophora.

\section{SUMMARY}

A study of the parasitic fauna, particulary the Gregarines, found in a troglobitic diplopod Typhloiulus bureschi Verhoeff, one of the Iulidae from bulgarian caves. Data are included about the morphology and taxonomy of the Eugregarines. There is a description of a new species, Stenophora typhloiuli. This species is compared with other species of the genus Stenophora.

\section{BIBLIOGRAPHIE}

GOLEMANSKY, V., Stenophora bulgarosomae n.sp. - une nouvelle grégarine (Gregarinida, Stenophoridae) de diplolode troglobie Bulgarosoma bureschi Verh. Zool. Ans. (in litt.).

GUÉORGUIEV, V., 1966 - Aperçu sur la faune cavernicole de la Bulgarie. Bull. Inst. Zool. \& Musée de Sofia, T. XXI, 157-184.

GUÉORGUIEV, V. et BERON, P., 1962 - Essai sur la faune cavernicole de Bulgarie. II. Résultats des recherches biospéologiques de 1961 à 1965. Bull. Inst. Zool. \& Musée de Sofia, 24, 151-212.

LEGER, L., et DOBOSCQ, O., 1963 - Recherches sur les Myriapodes de Corse et leurs parasites. Arch. Zool. Exp. et Gén., 4e série, 1, N 3, 307-359.

LÉGER, L., et DUBOSCQ, O., 1904 - Nouvelles recherches sur les Grégarines de l'épithélium intestinal des Trachéates. Arch. Protistk., 4, 335-383.

LIPPA, J.J., 1967 - Studies on Gregarines (Gregarinomorpha) of Arthropods in Poland. Acta Protozool., 5, N 8, 97-179.

STRASSER, K., 1966 - Über Diplopoden Bulgariens, Ann. Zoologici, 23, 12, 325-385.

WATSON, M.E., 1916 - Studies on Gregarines. Illinois Biol. Monographs., 2, N 3, 215-468. 